\title{
Zulassung nach Qualitätskriterien: einfach und wirksam
}

\author{
Jürg Schlup \\ Dr. med., Präsident der FMH
}

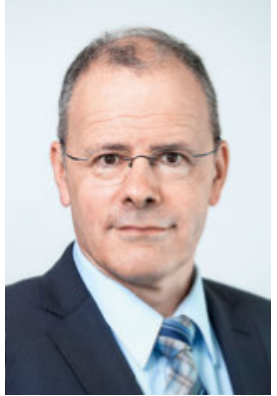

Weil das heutige System der Zulassungsbeschränkung für neue, praxisambulant tätige Ärzte und Ärztinnen am 30. Juni 2019 auslaufen wird, muss die Zukunft der Zulassung neu gestaltet werden. Die verschiedenen dazu im Parlament vertretenen Vorstellungen sind für Ärzteschaft und Bevölkerung bedeutsam, weil die Zulassung von Ärztinnen und Ärzten die Entwicklung unseres Gesundheitswesens entscheidend mitbestimmen kann. Dass diesmal eine definitive, dauerhafte Regelung beschlossen werden soll, verleiht den aktuellen Vorlagen nach 15 Jahren provisorischer Lösungen besonderes Gewicht.

\section{Zusätzliche Investition in Ausbildung ärzt-} lichen Nachwuchses - um diesem anschliessend eine Wartefrist zu verordnen?

So läuft noch bis zum 25. Oktober die Vernehmlassung zum bundesrätlichen Vorschlag einer administrativ aufwendigen Zulassungsregelung, die mit ihren vielen «kann»-Bestimmungen zudem recht undurchsichtig ist. Der Bundesrat will zukünftig nicht nur Voraussetzungen zu Bildungsabschlüssen und den «zur Qualität der Leistungserbringung notwendigen Strukturen» festlegen, er möchte auch unserem aufwendig ausgebildeten, noch knappen Nachwuchs nach abgeschlossenem Studium und Weiterbildung eine zweijährige Wartefrist auferlegen können. Zudem sieht er vor, die bisher durch dreijährige ärztliche Tätigkeit nachzuweisende Kenntnis unseres Gesundheitswesens neu durch eine theoretische Prüfung zu ersetzen. Ein zentrales Problem der Vorlage ist ausserdem die Stärkung der Interessenkonflikte der Kantone: Mit den ihnen zugesprochenen Befugnissen würden sie zukünftig sowohl ihre eigenen Spitalambulatorien als auch die praxisambulante Konkurrenz steuern. Auch für die Versicherer enthält der Gesetzesvorschlag eine Interessenkollision, denn sie dürften neu die Organisation bezeichnen, die über die Zulassung der Leistungserbringer entscheidet. Zudem sollen die Zulassungsvoraussetzungen nicht mehr auf Gesetzesstufe geregelt, sondern verordnet werden.

Weil der hier knapp skizzierte Vorschlag des Bundesrats nach Auffassung der nationalrätlichen Gesundheits- kommission «das System lediglich verfeinern will», ${ }^{1}$ fordert sie in einer Kommissionsinitiative zusätzlich mehr Einfluss der Krankenkassen: «Zulassungsverträge» der Ärzte mit Versicherern sollen zur Voraussetzung für eine Tätigkeit zulasten der OKP werden. Die ärztliche Berufsausübung würde zukünftig also nicht nur von den Kantonen, sondern massgeblich von den Krankenkassen bestimmt.

Nach Auffassung der FMH werden die hier dargestellten Bestrebungen, aufwendige Regulierungen und tiefgreifende Veränderungen voranzutreiben, weder der Situation im Gesundheitswesen noch der politischen Ausgangslage gerecht. Im Dezember 2015 scheiterte die Verstetigung des bis heute bewährten $\mathrm{Zu}$ lassungskriteriums «drei Jahre ärztliche Tätigkeit an anerkannter Weiterbildungsstätte in der Schweiz» haarscharf mit $96 \mathrm{zu} 97$ Stimmen. Verlangt ein Vorschlag, der den langen parlamentarischen Prozess erfolgreich passiert hat und am Ende an einer einzigen Stimme scheiterte, einen vollständigen Neuanfang? Der FMH scheint es unter diesen Voraussetzungen zielführender, die damals so knapp abgelehnte, bewährte Regelung mit gezielten Verbesserungen zum Erfolg zu führen. Darum schlagen wir drei einfach umsetzbare Qualitätskriterien zur Zulassungssteuerung vor: (1) Eine

Kantonale Interessenkonflikte werden verstärkt, wenn Kantone sowohl eigene Spitalambulatorien als auch konkurrierende Praxen steuern.

dreijährige ärztliche Tätigkeit in der für die Zulassung beantragten Fachdisziplin an einer anerkannten schweizerischen Weiterbildungsstätte. Dies würde die Kenntnis unseres Gesundheitswesens sicherstellen und wegen der indirekten Regulierung über das Stellenangebot auch eine bedarfsgerechte Verteilung der Fachdisziplinen gewährleisten. Verlangt man für eine Zulassung ausserdem die Überprüfung von (2) Fortbildung und (3) Sprachkompetenz, lassen sich mit Hilfe einer Zulassung nach Qualitätskriterien die Behandlungsqualität verbessern und die Patientensicherheit erhöhen. 